\title{
Tendencias actuales de la educación superior en Colombia*
}

\section{Current trends of higher education in Colombia}

John Jairo Gómez Ríos*** Henry Laverde Rojas**** Álvaro Díaz Niño****

Recibido: 20 de enero de 2016

Revisado: 22 de febrero de 2016

Aprobado: 8 de marzo de 2016

\section{Resumen}

El estudio de la economía en la actualidad se encuentra influenciado por diferentes tendencias que se originaron desde la última década del siglo XX. En este sentido, el objeto de análisis parte de lógicas claramente diferenciadas que dificultan la convergencia hacia un objetivo común entre el docente y el estudiante. El presente artículo se basa

* El presente artículo es producto de la investigación realizada para la Universidad Santo Tomás y financiada por la misma institución. Cómo citar este artículo: Gómez Ríos, J., Laverde Rojas, H. \& Díaz Niño, A. (2016). Tendencias actuales de la educación superior en Colombia. Revista CIFE, 18(28), 19-42. (DOI: http://dx.doi.org/10.15332/ s0124-3551.2016.0028.01

** Docente e investigador, Facultad de Economía, Universidad Santo Tomás.

*** Docente e investigador, Facultad de Economía, Universidad Santo Tomás.

**** Docente e investigador, Facultad de Economía, Universidad Santo Tomás. 
en un análisis de esta relación y el estudio de las prácticas pedagógicas que se generan en torno al estudio de la economía. Con base en información del Sistema Nacional de Información de la Educación Superior (SNIES), se estimó el impacto que el nivel de formación, las condiciones contractuales y los aspectos descriptivos de los docentes, tales como el género y el tipo de institución (pública o privada), tienen sobre los resultados de las pruebas Saber PRO como proxy de la calidad de la educación en Colombia. Los resultados muestran que, excepto el género de los docentes, todas las variables tienen un impacto estadísticamente significativo sobre la calidad de la educación en Colombia. También se realiza una revisión de la literatura acerca de esta temática y las tendencias que se originan en torno a su estudio.

Palabras clave: Educación, docencia, pedagogía, economía.

Clasificación JEL: A22

\section{Abstract}

The study of the economy at the present time is influenced by different trends that originated as of the last decade of 20th century. In this respect, the object of analysis is based on clearly differentiated logics that hinder convergence towards a common goal between the teacher and the student. This article is based on an analysis of this relation and the study of the pedagogical practices that are generated around the study of the economy. Based on information from the National Information System for Higher Education (SNIES), it estimated the impact that the level of training, contractual conditions and descriptive aspects of teachers, such as gender and type of institution (public or private), have on the Saber PRO test results as a proxy for the quality of education in Colombia. The results show that, except for the gender of teachers, all variables have a statistically significant impact on the quality of education in Colombia. There is also a review of the literature on this subject and the trends that originate around its study.

Keywords: Education, teaching, pedagogy, economics.

Classification JEL: A22 


\section{Introduction}

El estudio de la economía muestra diversas tendencias en el siglo XXI, que van desde el enfoque convencional derivado del modelo neoclásico, hasta el retorno al pensamiento keynesiano, desarrollado por los pensadores norteamericanos y europeos a raíz de la crisis del capitalismo en 2007-2008.

A finales del siglo XX y comienzos del XXI, se inició en Europa un proceso de autoevaluación y crítica surgido de algunas escuelas de economía francesas y estadounidenses que cuestionan profundamente los resultados del modelo neoliberal y su predominancia en la estructuración de los programas académicos en las universidades de alto prestigio a nivel mundial.

En este sentido, se debaten aspectos tales como el desfase de la enseñanza respecto a las realidades concretas de la sociedad o el uso desmesurado de las matemáticas, que lleva a la formalización de modelos que, más allá de la lógica, no contribuyen a explicar la realidad ni responden a los cuestionamientos de los debates económicos contemporáneos (de Botssieu et al., 2001).

De otro lado, se cuestiona la ausencia de reflexión y el dogmatismo, que no permiten la pluralidad de ideas y enfoques de la problemática económica, y se cree que el enfoque dominante presenta la verdad revelada en economía.

A pesar de la rigurosidad cuantitativa que ha adquirido el estudio de la economía desde los comienzos de su formalización, no debe olvidarse que sigue teniendo como objeto de estudio fenómenos claramente sociales. En este sentido, el proceso de enseñanza en economía es particularmente interesante como ejercicio pedagógico, ya que abarca dos niveles de formación: el primero de ellos hace una aproximación entre la ciencia económica y las ciencias formales (p. ej.: las matemáticas), pues mediante el uso de la lógica formal busca llegar a inferencias que den soporte a planteamientos e hipótesis; el segundo nivel se relaciona con las ciencias sociales (administración, antropología, ciencia política) porque busca ocuparse de aspectos de los seres humanos y su entorno mediante el uso de instrumentos de tipo argumentativo y analítico (Tobón, 2008).

El presente documento busca hacer una revisión de la literatura reciente en torno al estudio y método de la enseñanza en economía, tomando como principio el argumento anteriormente expuesto. Se encuentra organizado como sigue: en la segunda sección se describen los principales postulados hechos en torno al estudio de la economía en los últimos tiempos; posteriormente, se hace una revisión de la literatura reciente sobre el tema, tanto a nivel local como internacional; la siguiente sección presenta los hechos estilizados; luego, se discuten los principales hallazgos producto de las estimaciones econométricas; en la última sección se concluye. 


\section{El proceso de enseñanza: desde la pedagogía hacia la economía}

El proceso enseñanza-aprendizaje parte de una correspondencia social compleja: la relación docente-estudiante. Esta relación parte de un elemento esencial de la conducta humana, a saber, el deseo constante por adquirir conocimiento.

En este sentido, algunos autores (Quintero y Giraldo, 2006) señalan que la educación es una parte del ser humano y consideran que la relación entre el docente y el estudiante (o discípulo) parte de una complementariedad según la cual el primero es poseedor de un conocimiento previo y de una vocación para transmitirlo a su estudiante. Por su parte, este último se encuentra dispuesto a recibirlo y adoptarlo como parte de su proyecto de vida.

No obstante, en lo referente al estudio de la economía, el objeto de análisis parte de lógicas claramente diferenciadas que dificultan la convergencia de estos dos actores hacia un objetivo común.

Por un lado, la enseñanza de la economía parte de un método de análisis transversal a todas las ciencias: la teoría. La teoría económica brinda un instrumento de análisis para explicar los fenómenos de la sociedad de una manera simple y abstracta. No explica fielmente la realidad, sino que, a partir de modelos (abstracciones de la realidad), analiza los fenómenos antes mencionados. No obstante, debido a que las decisiones de los individuos no obedecen a lógicas inmutables y están enmarcadas dentro de fenómenos cambiantes, el estudio de la economía se torna complejo y hace que decisiones individuales claras y espontáneas, tales como el ahorro y la inversión, entre otras, no resulten fácilmente explicables.

Esta situación hace que el estudiante, en algunas ocasiones, no pueda encontrar soluciones reales a fenómenos reales relacionados con su entorno, por ejemplo, el desempleo, la inflación, la pobreza y las crisis, entre otros, que le resultan difíciles de asimilar y aceptar en un entorno más exigente y competitivo. A esta situación se le suma la necesidad de ser un profesional capaz de dar respuesta clara a estos fenómenos, tal como lo exige la sociedad (Tobón, 2008).

No obstante, esta división no es reciente, pues se remonta a los comienzos del estudio de la economía como ciencia. En la actualidad, los estudiantes reclaman respuestas concretas de la ciencia económica a problemas económicos dentro de un contexto más social, partiendo del principio de que el mundo se encuentra en un entorno económico cada vez más globalizado.

A pesar de lo anterior, dichas circunstancias no han representado un impedimento para que la ciencia económica haya evolucionado y se haya impuesto como una ciencia social rigurosa, que puede brindar explicaciones y argumentos de gran importancia para los 
fenómenos económicos de la sociedad actual, lo cual ha brindado un elemento de base para la toma de decisiones en materia de política económica.

Por lo tanto, la misión más importante del docente en la actualidad es concientizar a los estudiantes acerca de los aportes realizados por la ciencia económica a la explicación de algunos fenómenos de la realidad, tal como es el caso de las crisis ocurridas en los últimos años, algunas de las cuales fueron pronosticadas con gran precisión por modelos teóricos y empíricos elaborados con anterioridad a su ocurrencia. Así mismo, es importante que el estudiante sea consciente del interés permanente de la ciencia económica por ir perfeccionando la teoría y los modelos de tal manera que se puedan enfrentar los fenómenos reales con instrumentos cada vez más precisos y eficaces, en un proceso constante de transformación, de forma tal que el estudiante apropie la importancia de una ciencia útil para su sociedad.

Actualmente, las escuelas (facultades) de economía se enfocan en la incorporación de elementos de aplicación en sus contenidos curriculares, de tal forma que los estudiantes puedan contar con elementos de contexto más precisos que les permitan explicar fenómenos económicos reales con base en la teoría. La incorporación de temas de economía agregada - tales como cuentas nacionales, inflación, desempleo, pobreza, distribución del ingreso, desarrollo económico, tasas de cambio, entre otros- ${ }^{-}$y temas de comportamiento de los agentes - por ejemplo, análisis de estructuras de mercados monopólicos, costos de producción y costos de oportunidad- ha brindado a los estudiantes elementos de estudio bastante consistentes con la realidad económica de su entorno. La estadística, por su parte, ha dado un aporte instrumental fundamental a la economía por medio del análisis econométrico, mediante el cual, el estudiante puede aplicar conjuntamente los conceptos adquiridos en las teorías micro y macroeconómicas. De la misma manera, la incorporación de espacios académicos en los que se analizan temas de actualidad, tales como coyuntura económica, o economía colombiana, sirve de elemento de contexto para aproximar al estudiante mucho más con su entorno.

Por su parte, las finanzas representan un componente de énfasis aplicado altamente significativo mediante la formulación y evaluación de proyectos y el análisis de mercados de capitales.

Por tal razón, la labor del docente en la formación del economista es de gran relevancia, ya que debe concientizar al estudiante sobre la importancia que la teoría tiene para la explicación de la realidad y los múltiples instrumentos que posee para tal fin.

\subsection{Formación en economía}

La formación del economista se orienta hacia la consecución de un conocimiento amplio, que ha de estar alineado con las realidades socioeconómicas de su entorno y del entorno global. 
La tendencia actual de la carrera de Economía en América Latina se caracteriza por dirigirse hacia el diseño de programas de corta duración, con tiempos de graduación de entre 4 y 5 años, lo que hace unos años implicaba, en promedio, un año más de preparación. La mayoría de las instituciones de educación superior cuentan con asignaturas orientadas al apoyo instrumental del análisis económico y áreas de formación básica propias de las ciencias económicas. El componente de formación complementaria comprende, en el 75 \% de las universidades, las áreas de finanzas, contabilidad y evaluación de proyectos. Los ciclos de profundización -compuestos por asignaturas tales como administración, técnicas de investigación y derecho- se encuentran en cerca de la mitad de los programas, mientras que un menor porcentaje de las instituciones cuenta con profundización en un segundo idioma y en teoría de juegos.

Esta estructura observada en las instituciones de educación superior de la región, concentrada en materias con un fuerte componente cuantitativo (matemáticas, estadística) y con un acentuado componente económico, contrasta con las estructuras menos especializadas de los departamentos de Economía de algunas instituciones norteamericanas: resalta la importancia de una formación básica más amplia en humanidades, historia y política, con un desarrollo de destrezas en lectura, escritura y expresión oral (Lora y Ñopo, 2009).

El contar con diversos campos de especialización ofrece a los estudiantes la posibilidad de alcanzar un mejor grado de preparación con miras a enfrentar el mundo laboral. Para el caso latinoamericano, puede observarse que una de las principales áreas de especialización es la de economía empresarial y administración y, en menor medida, economía aplicada y economía política y social. De esta manera, el futuro profesional responde a la demanda del mercado, en el que las instituciones financieras, las firmas consultoras y las oficinas de planeación (estatales y privadas) buscan economistas con amplias competencias en la toma de decisiones en el campo empresarial y en la formulación de políticas sociales y económicas.

Por otra parte, las instituciones de educación superior de la región orientan sus actividades de extensión hacia el ámbito empresarial y social. El modelo económico mundial predominante está dirigido a que gran parte de las universidades encaucen sus esfuerzos en actividades de extensión que puedan responder a las necesidades del entorno donde estas se llevan a cabo; en este sentido, se marca una tendencia hacia el desarrollo de esta función, para que se oriente hacia el enfoque empresarial, en el cual, la institución se concibe como una empresa que interactúa con el mercado y que responde a las necesidades del sector productivo. Esta concepción se aparta del modelo tradicional, que parte del principio según el cual la universidad es fuente de conocimiento, y también del modelo de desarrollo integral, para el que la universidad asume una función social al contribuir en el mejoramiento de la calidad de vida de la sociedad (Ortiz-Riaga y Morales-Rubiano, 2011). 


\section{Revisión de la literatura}

Desde hace varios años, se han venido desarrollando algunos escritos que cuestionan y proponen alternativas de análisis en torno a la educación superior y al estudio de la economía. En la presente sección se elabora una revisión de la literatura relacionada con pedagogía universitaria, en general, y en economía, de tal manera que se pueda tener un espectro amplio alrededor del tema y establecer puntos de partida para futuras discusiones.

\subsection{Docencia universitaria}

La educación superior del siglo XXI se encuentra enmarcada en torno al desarrollo de dos grandes tendencias: la globalización y la llamada "sociedad del conocimiento".

Los fenómenos ocurridos durante la última década del siglo XX -tales como la separación de la Unión Soviética, la caída del Muro de Berlín y el resurgimiento de Estados subyugados debido a los designios políticos del antiguo bloque socialista- son aspectos que han marcado el orden económico, social y político de las dos primeras décadas del presente siglo. Dichas circunstancias han traído lo que podría clasificarse como "ganadores" y "perdedores". En el primer grupo se encuentran aquellas potencias que han detentado por muchos años la hegemonía capitalista y aquellas que han decidido dar un giro total a su economía, pasando de ser planificadas a convertirse en completas economías de mercado. Esta situación ha permitido que el avance de la sociedad se dé en términos desiguales y ha hecho que las economías consideradas como más "débiles" terminen siendo las menos favorecidas en la competencia de las grandes economías por mantener e incrementar su poderío económico; este puede ser el caso de quienes conforman el segundo grupo.

Pero este avance de la sociedad ha permitido que también se presente un acelerado avance tecnológico. Tal y como lo menciona Durán (2006), dicho avance acelera así mismo la consecución de la información, que a su vez acelera "la homogeneización de las costumbres, en detrimento de las culturas locales y la producción nacional" (Peñate, 1998, p. 4, tomado de Durán, 2006), situación que ha posibilitado el surgimiento de fenómenos de desigualdad, los cuales, a su vez, desencadenan mayores niveles de pobreza.

La creación de nuevos bloques y acuerdos comerciales (tratados de libre comercio) ha obligado a las sociedades modernas a volverse más competitivas con el propósito de no rezagarse ante los nuevos avances y evitar ser excluidas de este nuevo entorno. Esta situación ha dado origen a una "revolución pedagógica" que toma como instrumento fundamental las nuevas tecnologías de la información y la comunicación (TIC) y que ha llevado a la educación superior a una era de transnacionalización de la información, lo 
cual representa un reto en la denominada "era global", que obliga a las instituciones a hacer una revisión de sus planes de currículo, de estudio y a implementar instrumentos pedagógicos para tal fin (Durán, 2006).

No obstante, todo lo anterior tendrá poca trascendencia si no viene acompañado de políticas que garanticen la inclusión de todos los sectores de la sociedad (en especial, las menos favorecidas) a un sistema de educación que permita mejorar las condiciones de desigualdad existentes en la actualidad. La mejora en los sistemas educativos debe venir acompañada de políticas gubernamentales que garanticen la inclusión social y el mejoramiento del capital humano, a pesar de que esta no es la única solución al problema de subdesarrollo que enfrentan nuestras sociedades latinoamericanas. Es decir, no se puede subyugar el problema de desigualdad y miseria existente en el continente a la deficiente educación cuando existen problemas institucionales de mayor impacto y relevancia, como es el caso de la corrupción de las élites (Dieterich, 1996).

\subsection{La enseñanza en economía}

En concordancia con lo anterior, la enseñanza de la economía debe estar circunscrita a los mismos preceptos planteados por la pedagogía universitaria en general, es decir, debe estar apoyada en el uso de diversas ayudas pedagógicas (tales como las TIC), pero más importante aún, debe responder a las necesidades del entorno y a la problemática socioeconómica existente en la actualidad. En este sentido, es necesaria la creación de un modelo pedagógico sobre la enseñanza de la economía.

De acuerdo con Tobón (2008), existe alguna literatura reciente acerca de la enseñanza de la economía, pero esta solamente se circunscribe al planteamiento y a la recolección de algunos aspectos pedagógicos y didácticos, ya que la enseñanza en economía se entiende como la modificación de contenidos formativos del pregrado en economía y ajustes a los temas investigativos. Por tal razón, el autor propone la creación de un modelo pedagógico para la enseñanza en economía basado en cuatro esferas del proceso enseñanza-aprendizaje: i) cátedra magistral del profesor, ii) trabajo académico en el aula, iii) trabajo académico individual y grupal fuera del aula y iv) asesoría.

Algunos autores, por su parte, han planteado que una de las falencias existentes en la enseñanza de la economía radica en la incapacidad de incorporar problemáticas que son inherentes a la condición de subdesarrollo de nuestra sociedad. En este sentido, SfeirYounis (2009) enfatiza en la necesidad de un cambio en materia de educación ambiental, enfocado especialmente hacia el área de la economía ambiental, pues considera que la principal causa de la destrucción de nuestro capital natural radica en un conjunto de patrones y prácticas económicos que afectan el progreso de nuestra sociedad e impactan otras esferas de carácter económico. 


\subsection{El economista en el ámbito laboral}

En la mayoría de las economías existe una estrecha relación entre los trabajos desempeñados por los trabajadores y su nivel de formación. De hecho, se considera que, en la mayoría de los casos, la oferta de mano de obra calificada (profesionales recién egresados) debe estar acorde con las plazas laborales ocupadas. No obstante, dicha relación no es totalmente clara y en algunas ocasiones se observa sobreoferta de mano de obra en ciertas áreas de conocimiento, lo cual genera ineficiencias en la asignación de recursos. De hecho, muchos empleos están definidos en función de unos requerimientos mínimos en cuanto a formación, lo cual requiere una demanda específica de profesionales. En el ámbito de la economía, la demanda de puestos de trabajo se distribuye uniformemente entre el sector público y el privado, pero el número de plazas disponibles hace que se presente una sobreoferta de mano de obra que la obliga a ubicarse en plazas que no responden a las competencias específicas ofrecidas por el economista. En este sentido, Salas (2004) elaboró un estudio para el caso español, según el cual los profesionales de diversas áreas (entre las cuales está la economía) se encuentran, en su primer empleo, desempeñando actividades acordes con su formación, lo cual se refuerza cuando el profesional cuenta con cierta experiencia laboral.

Por su parte, Malamud y Pop-Eleches (2008) elaboraron un estudio usando datos de la encuesta de hogares y del censo para Rumania, durante el período 1992-2002. El estudio examinó el beneficio relativo de los niveles de educación y del entrenamiento vocacional en dicho país, teniendo en cuenta sus mejoras tecnológicas y los cambios institucionales ocasionados por la transición de un sistema comunista a una economía de mercado. Tomando en consideración la reforma educativa aplicada en 1973, que cambió una gran proporción de estudiantes de formación técnica a educación profesional, los resultados muestran que la mano de obra calificada beneficiada con la reforma educativa fue significativamente menos propensa a desempeñar actividades manuales que aquellas personas que no se beneficiaron con la reforma.

Gazzola y Didriksson (2008) realizaron una recopilación de escritos de varios autores en torno a las tendencias de la educación superior en América Latina y el Caribe, en la que se integran diferentes temas en el campo de la educación superior enfocados desde una perspectiva regional. El propósito de dicho documento consiste en identificar escenarios posibles y tendencias de largo plazo en la educación superior en la región desde una visión prospectiva. De esta manera, se propone presentar la situación de diferentes aspectos de la educación superior por medio de indicadores a partir de los cuales se puedan construir bases de datos a nivel nacional comparables para la posterior elaboración de una base regional, similar a un atlas construido por capas, etapas o conjuntos de indicadores, que hagan posible un acercamiento gradual a la realidad de la educación superior en la región; el proyecto resultaría en fotografias sucesivas en cortes históricos simultáneos. 
En cuanto al estudio de la economía, se destaca el documento Discusión francesa sobre la enseñanza de la economía, elaborado por varios estudiantes de Economía de distintas instituciones de educación superior del país Galo y traducido al español por Cataño (2001). En dicho documento, los estudiantes hacen una crítica sobre los principales postulados actuales realizados principalmente por la economía clásica; también se exponen los postulados realizados por los profesores de Economía de las mismas instituciones en torno a la misma problemática, los cuales concuerdan en algunos aspectos, pero discrepan en otros.

En esta misma línea se desarrollaron, especialmente en Europa, una serie de documentos y manifiestos que ponían en entredicho los métodos y las teorías adoptados por las principales escuelas de economía y que expresaban el sentir de los estudiantes de las escuelas de economía sobre la enseñanza que estaban recibiendo ${ }^{1}$.

Se destacan también los documentos de Katz (2001), quien elaboró una crítica a los economistas ortodoxos centrando su análisis en la concepción de que la economía es una ciencia que se encuentra, en algunos casos, a merced de clases dominantes que justifican el dominio de mercados y legitiman la desigualdad con base en la propagación de teorías naturalistas y mecanicistas, en el marco de una ortodoxia dominada por la hegemonía norteamericana.

Revisando la literatura del lado americano, Becker y Watts (2001), basados en fuentes primarias de información (encuesta directa a economistas académicos), realizaron un análisis acerca de los métodos de enseñanza en economía para los años 1995 y 2000 en cuatro tipos de programa de pregrado para los Estados Unidos. A partir de la comparación de los resultados encontrados en cada año, los autores hallaron que, a pesar del marcado interés por mejorar las técnicas, los métodos de enseñanza en general en esos cursos han cambiado muy poco y siguen dominados por el esquema chalk and talk, es decir, clases magistrales.

Para el caso de nuestro continente, el documento de trabajo elaborado por Lora y Ñopo (2009) presenta una visión comparada de la formación de economistas para cinco países de América Latina. Teniendo en cuenta aspectos como currículo, libros de texto comúnmente utilizados, dedicación de los profesores, métodos de enseñanza y uso de tecnologías y herramientas computacionales, los autores aplicaron una encuesta a estudiantes de esta carrera para diseñar su perfil socioeconómico e indagar acerca de sus actitudes y opiniones. Dentro de las regularidades encontradas, es de resaltar la falta de conexión entre las expectativas de los estudiantes sobre las demandas de los mercados de trabajo y las demandas efectivas de economistas por sectores de empleo.

1 Se destacan el Manifiesto de Bilbao y el Manifiesto de estudiantes por una economía crítica. 
Por su parte, Ahumada y Butler (2009) examinaron las características de los programas de estudio y los atributos y percepciones de los estudiantes de nivel de licenciatura en seis de las principales facultades de Economía de México, así como la evolución, la inserción laboral y las percepciones de los economistas en el período 2000-2005. Los autores notaron tendencias que incluyen una pequeña disminución de las matrículas en carreras de Economía, un aumento del número de economistas empleados en el sector privado, una constante participación de las mujeres de aproximadamente la tercera parte de los ingresados en programas de Economía y un paulatino, pero innegable incremento del uso de Internet y otras herramientas de computación.

Rozenwurcel, Bezchinsky y Rodríguez (2009) reseñaron la enseñanza de economía en Argentina, empezando por los antecedentes relevantes y las principales características del sistema universitario argentino, antes de abordarse el tema principal desde sus orígenes hasta la evolución de los últimos años. Posteriormente trataron directamente al análisis de la enseñanza en las tres universidades seleccionadas (Universidad de Buenos Aires, Universidad Nacional de Córdoba y Universidad Torcuato Di Tella), utilizando para ello los resultados de una encuesta de opinión a estudiantes e información recabada mediante entrevistas con informantes clave. Concluyeron que, aunque la enseñanza de la economía en Argentina se encuentra mayormente en buenas condiciones, existen áreas específicas que presentan oportunidades para mejoramiento.

Para el caso colombiano, se destaca el documento de Sarmiento y Silva (2014), quienes elaboraron un estudio sobre las características de la educación recibida por los estudiantes de Economía en Colombia, mediante un análisis de las condiciones socioeconómicas, las preferencias y el desenvolvimiento dentro de la carrera, así como del marco institucional y académico a partir del análisis estadístico de una muestra representativa cercana al $90 \%$ de los programas con registro calificado para el país.

\section{Hechos estilizados de la educación superior en Colombia}

La formación superior en Colombia ha sufrido una serie de transformaciones de toda índole, con especial énfasis en elaboración de procesos empíricos, implementación de herramientas tecnológicas y comunicaciones, lo que ha resultado en una mejora de los procesos de enseñanza-aprendizaje. Sin embargo, existen todavía muchos aspectos por mejorar que arrojan ciertos niveles de rezago en cuanto a la calidad de los procesos académicos. 
En este sentido, el talento humano se convierte en el factor determinante para alcanzar estándares de calidad que permitan que la educación superior en Colombia alcance niveles competitivos acordes y comparables a nivel internacional.

Se presenta a continuación un análisis de diversos factores que caracterizan al capital humano docente de las instituciones de educación superior en Colombia. Para esto, se tomó como referencia la información disponible en el Sistema Nacional de Información de la Educación Superior (SNIES), que recopila la información suministrada por la mayoría de las instituciones de educación superior en el país. La muestra incluye instituciones de carácter público y privado distribuidas en proporciones similares (privadas $-55 \%$, públicas $-45 \%$ ).

Figura 1. Tipo de universidad

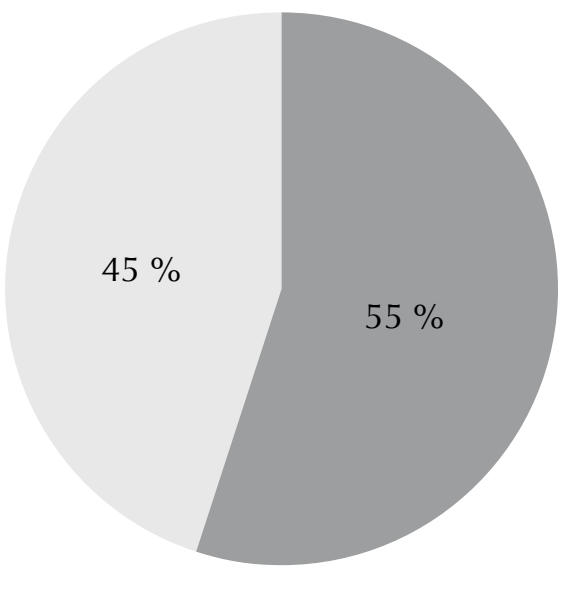

Públicas Privadas

Fuente: elaboración propia con base en datos de SNIES (2014).

\subsection{Nivel de formación docente}

El nivel de formación de los docentes es un elemento crucial con miras a alcanzar altos estándares de calidad académica, ya que, mientras mayor sea su nivel educativo, mayores serán las competencias que se van a desarrollar por parte de ellos en aspectos tales como docencia, investigación y proyección social. Con base en la información proveniente del Sistema Nacional de Información de la Educación Superior (SNIES), se observa que la mayoría de los profesores universitarios tiene nivel de maestría (30 \%), seguido de formación universitaria y especialización (29 \%), mientras que solo el $7 \%$ cuenta con formación doctoral y posdoctoral, situación preocupante en términos de calidad educativa, pues esto puede mostrar evidencia acerca de la preocupación de las instituciones por reforzar sus procesos académicos de docencia, rezagando la función 
sustantiva de la investigación, elemento de relevante dentro de las funciones institucionales, puesto que por medio de la investigación se puede generar conocimiento y potenciar el desarrollo en general.

Figura 2. Formación profesional docente

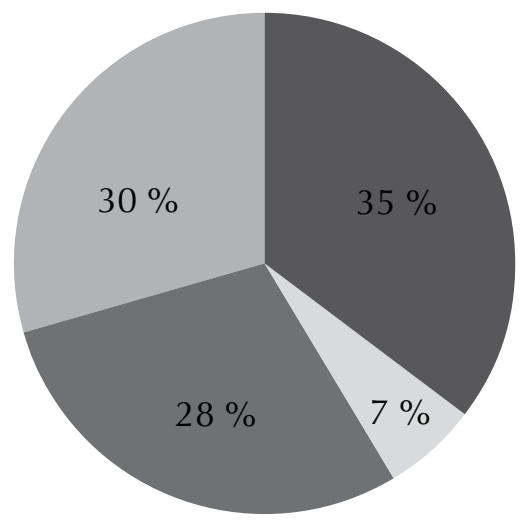

Doctorado Posdoctorado Maestría $\square$ Profesional $\square$ Otros

Fuente: elaboración propia con base en datos de SNIES (2014).

\subsection{Tiempo de dedicación}

Otro aspecto que siempre se ha considerado como tema de debate en la educación superior es el relacionado con la dedicación de los docentes a las funciones sustantivas de la educación. En este sentido, es de esperar que un docente esté dedicado de manera exclusiva a sus labores académicas en una sola institución.

No obstante, el desarrollo de la actividad docente ha estado demarcado por una fuerte disyuntiva basada en la productividad versus la remuneración. Se tiende a creer que la remuneración de los docentes en educación superior no está compensada con su nivel de formación ni con las actividades realizadas. Esto conlleva una situación que va en detrimento de la calidad académica: se hacen comunes los casos de educadores vinculados a varias instituciones educativas, sin tener en cuenta sus capacidades laborales. Esto plantea un gran interrogante en torno al tema de la educación superior en Colombia: ¿Es la remuneración de los docentes suficientemente alta como para evitar fenómenos de movilidad académica voluntaria entre instituciones?

El ideal para una institución de educación superior es poder contar con docentes de dedicación suficiente para aportar a su proceso de crecimiento tanto a nivel interno como global. De acuerdo con la información del SNIES, las instituciones de la muestra 
cuentan, en su mayoría, con docentes catedráticos dentro de su planta (50\%), de medio tiempo $(10 \%)$ y solo el $29 \%$ son de tiempo completo.

Figura 3. Tiempo de dedicación

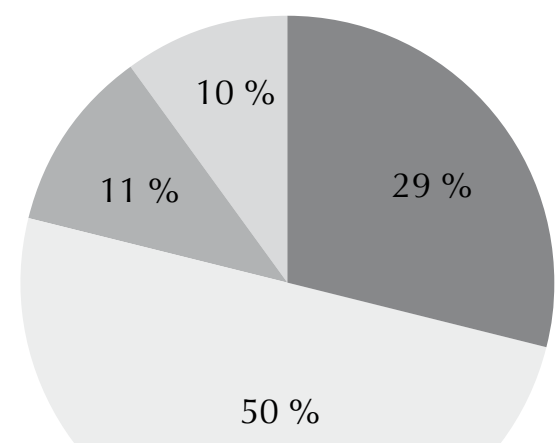

Catedra Medio tiempo Parcial $\square$ Tiempo completo

\subsection{Tipo de contratación}

Una de las posibles explicaciones por las cuales existe un gran número de docentes con vinculaciones a diferentes instituciones de educación superior puede estar relacionada con el tipo de contratación. En concordancia con lo anterior, también es de esperar que el tipo de vinculación de los docentes permita alcanzar estándares de calidad lo bastante altos como para ser lo suficientemente competitivos a nivel regional y global.

Con los resultados de la información extraída de la muestra de instituciones, se puede observar que la mayoría de los docentes cuenta con contrataciones distintas a contratos a término fijo o término indefinido, en contraste con el $16 \%$ que cuenta con contrato a término fijo; solo un $12 \%$ tiene contrato a término indefinido, lo que evidencia las condiciones precarias en sus contratos laborales. Esto, sumado al tipo de dedicación, muestra que el tipo de contratación puede incidir significativamente en la calidad educativa de los planteles educativos, por cuanto los profesores, en busca de mejores condiciones, tienen que encontrar otras fuentes de ingresos, situación que incide sobre su rendimiento en las universidades. 
Figura 4. Tipo de contrato

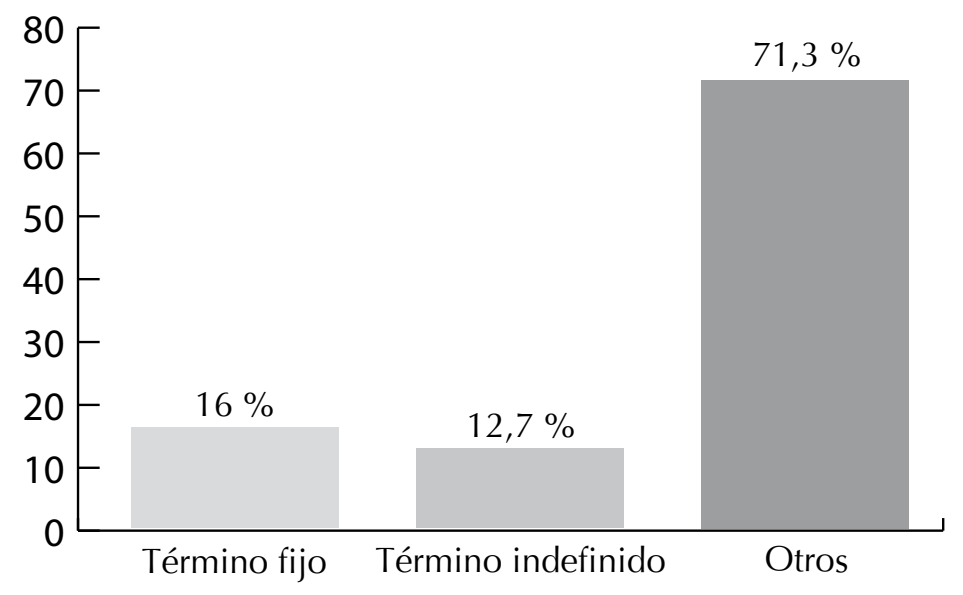

Fuente: elaboración propia con base en datos de SNIES (2014).

\subsection{Capital humano por género}

El análisis de la distribución del género puede dar luces de fenómenos discriminatorios en las instituciones de educación superior. Como ha sido reportado en varios estudios, las brechas salariales y de oportunidades para las mujeres en comparación con los hombres pueden explicarse por fenómenos culturales y machistas de la sociedad colombiana; esto también puede extenderse al ámbito académico. De acuerdo con los datos del Sistema Nacional de Información de la Educación Superior - SNIES, se observa que en la mayoría de las instituciones se contrata en su mayoría a hombres (63\%), lo que evidencia el sesgo y la segregación mencionados.

\subsection{Internacionalización de docentes}

Ante un mundo cada vez más globalizado y más integrado, se hace necesario para las instituciones de educación superior compartir experiencias en términos de intercambios, convenios de internacionalización, transferencia de conocimientos, etc. En este sentido, un aspecto importante es la oportunidad de que profesores internacionales trabajen en las instituciones colombinas con el ánimo de compartir con ellos su conocimiento y experiencias.

De acuerdo con los datos, los espacios para la internacionalización para las universidades colombianas aún siguen siendo muy insignificantes, pues los profesores extranjeros que trabajan en las universidades colombianas representan apenas el $1.3 \%$. 


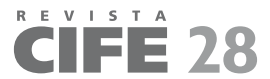

ISSN: 0124-3551 / Año 18, No 28 / enero-junio / pp. 19-42

Figura 5. Profesores por género

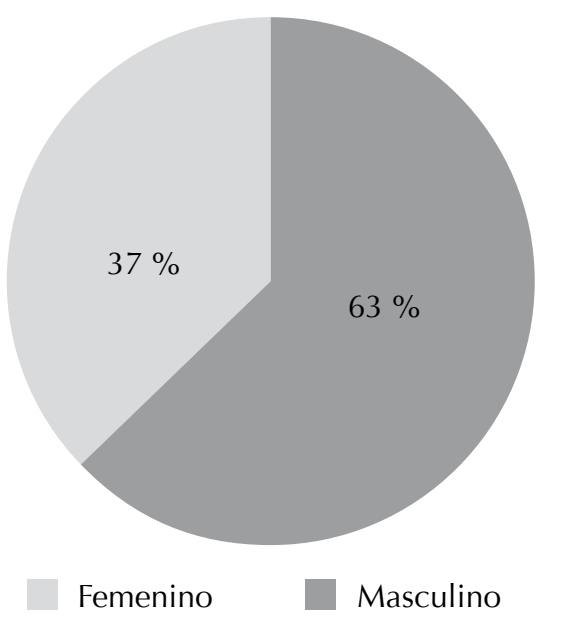

Fuente: elaboración propia con base en datos de SNIES (2014).

Figura 6. Profesores extranjeros

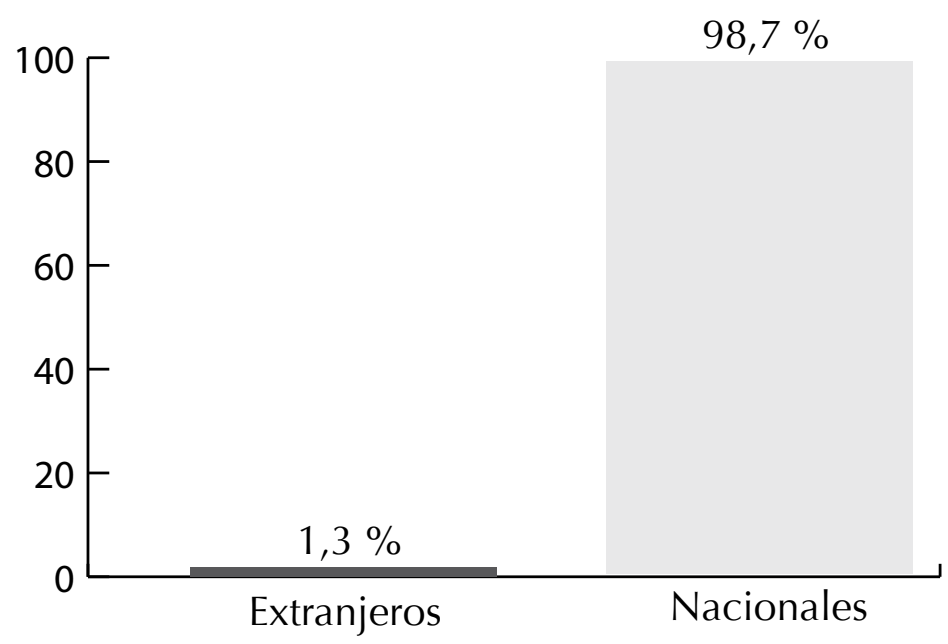

Fuente: elaboración propia con base en datos de SNIES (2014). 


\section{Análisis econométrico de la calidad de la educación superior en Colombia}

Con el ánimo de dar evidencia empírica sobre la incidencia que tienen las diferentes variables estudiadas en el apartado anterior sobre la calidad educativa, en esta parte se plantea un modelo econométrico de sección cruzada para una muestra de 112 universidades a nivel nacional. El modelo que se propone está planteado de la siguiente manera:

$$
y_{i}=X^{\prime} \beta+e_{i}
$$

En la fórmula, $y_{i}$ es una variable que caracteriza la calidad educativa, $X^{\prime}$ es un vector de variables que inciden sobre la variable dependiente y $e_{i}$ es el término de error. Los datos para la estimación de los parámetros del modelo provienen de los resultados de estado de la Prueba Saber Pro y el SNIES, de la siguiente manera:

$y_{i}$ : Puntaje de los resultados de estado de la prueba Saber Pro de la universidad $i$.

$x_{1:}:$ Variable binaria que toma el valor de 1 si es universidad pública, 0 en cualquier otro caso.

$x_{2}:$ Formación académica de los docentes de la universidad $i$.

$x_{3 i}:$ Variable binaria que toma el valor de 1 si los docentes de la institución educativa tienen un tipo de contrato de tiempo completo, 0 en cualquier otro caso.

$x_{4 i}:$ Variable binaria que toma el valor de 1 si los docentes cuentan con contratos de término fijo o término indefinido, 0 en cualquier otro caso.

$x_{5 i}:$ Variable binaria que toma el valor de 1 si los docentes son de género masculino, 0 en cualquier otro caso.

$x_{6 i}:$ Variable binaria que toma el valor de 1 si los docentes son extranjeros, 0 en cualquier otro caso.

Las estimaciones se realizan mediante regresiones de mínimos cuadrados ordinarios (MCO) y errores robustos.

\subsection{Resultados}

La tabla 1 muestra los resultados de las estimaciones econométricas. La variable dependiente está conformada por los resultados de estado de la prueba Saber Pro de la universidad i y las variables dependientes que se incluyen son las 5 dimensiones estudiadas anteriormente, en las que se quiere evaluar cuáles componentes son más determinantes para la educación colombiana. Las variables dependientes tomadas en cuenta para este 
análisis están divididas por bloques. En primer lugar, se incluyen variables relacionas con la formación de los docentes y con el tipo de universidad. Este modelo muestra que las variables tienen los signos esperados y que en todos los casos son altamente significativas: un mayor nivel de formación de los docentes y pertenecer a una universidad pública (esta última variable es una dummy que toma el valor de 1 si es una universidad pública, 0 en cualquier caso) aumentan las probabilidades de obtener mejores resultados en las pruebas de Estado. Como era de esperarse, estas dos variables se convierten en factores importantes para la calidad educativa.

Con el ánimo observar otros componentes y hacer un análisis de robustez, en las siguientes columnas de la tabla se presentan diferentes modelos agregando paulatinamente nuevos bloques al modelo base. En primer lugar, el modelo 2 incorpora dos variables relacionadas con las condiciones laborales de los docentes universitarios, el tiempo de dedicación y el tipo de contrato. Estas dos variables explican significativamente los resultados de las pruebas de Estado. En cuanto a la robustez del modelo, la inclusión de estas nuevas variables no parece afectar el comportamiento de las variables incluidas en el modelo 1, ni en significancia estadística ni en impacto sobre la variable dependiente.

Tabla 1. Regresión para la calidad educativa en Colombia 2014

\begin{tabular}{|l|c|c|c|c|}
\hline & Modelo 1 & Modelo 2 & Modelo 3 & Modelo 4 \\
\hline Formación de los docentes & $0.007^{* * *}$ & $0.007^{* * *}$ & $0.007^{* * *}$ & $0.006^{* * *}$ \\
\hline Tipo de universidad & $(0.001)$ & $(0.001)$ & $(0.001)$ & $(0.001)$ \\
\hline Dedicación laboral & $0.016^{* * *}$ & 0.010 & $0.014^{* * *}$ & 0.005 \\
\hline Tipo de contrato & $(0.016)$ & $(0.006)$ & $(0.006)$ & $(0.006)$ \\
\hline Género de los profesores & & $0.098^{* * *}$ & & $0.087 * * *$ \\
\hline Nacionalidad & & $(0.014)$ & & $(0.014)$ \\
\hline Constante & & $0.344^{* * *}$ & & $0.336^{* * *}$ \\
\hline R2 & & $(0.012)$ & & $(0.011)$ \\
\hline Número de observaciones & & & 0.004 & 0.006 \\
\hline
\end{tabular}


El modelo 3 realiza el mismo análisis del modelo 2, pero incorpora dos variables relacionadas con el género y la nacionalidad de los profesores. Aunque ante este nuevo modelo, sorprendentemente el género no es significativo estadísticamente, la nacionalidad se muestra importante para explicar la calidad educativa en Colombia. Nuevamente, las variables del modelo 1 parecen inertes ante este nuevo contexto, excepto la variable tipo de universidad, cuya significancia estadística se reduce en relación con el modelo 1 .

Finalmente, el modelo 4 agrega simultáneamente todas las variables discutidas con anterioridad. Como se observa, todas las variables resultan altamente significativas, con un fuerte impacto, excepto las relacionadas con el tipo de universidad. Los análisis de robustez muestran que, ante las diferentes especificaciones, estas variables evidencian un buen comportamiento: las variables afines son formación educativa, dedicación laboral, tipo de contrato, género y nacionalidad. Como análisis de robustez adicional, estos mismos modelos son realizados para los años 2015 y 2016 (mostrados en las tablas 2 y 3). Los resultados generales se mantienen para estos años y evidencian un alto grado de robustez en los resultados encontrados en la tabla 1.

Tabla 2. Regresión para la calidad educativa en Colombia 2015

\begin{tabular}{|c|c|c|c|c|}
\hline & Modelo 1 & Modelo 2 & Modelo 3 & Modelo 4 \\
\hline Formación de los docentes & $\begin{array}{c}0.007 * * * \\
(0.001)\end{array}$ & $\begin{array}{c}0.006 * * * \\
(0.001)\end{array}$ & $\begin{array}{c}0.006^{* * * *} \\
(0.001)\end{array}$ & $\begin{array}{c}0.004 * * * \\
(0.001)\end{array}$ \\
\hline Tipo de universidad & $\begin{array}{c}0.034^{* * * *} \\
(0.008)\end{array}$ & $\begin{array}{c}0.028 * * * \\
(0.008)\end{array}$ & $\begin{array}{c}0.025^{* * * *} \\
(0.007)\end{array}$ & $\begin{array}{c}0.016 * * \\
(0.007)\end{array}$ \\
\hline Dedicación laboral & & $\begin{array}{c}0.056^{* * *} \\
(0.014)\end{array}$ & & $\begin{array}{c}0.042^{* * * *} \\
(0.012)\end{array}$ \\
\hline Tipo de contrato & & $\begin{array}{c}0.411 * * * \\
(0.011)\end{array}$ & & $\begin{array}{c}0.375^{* * * *} \\
(0.010)\end{array}$ \\
\hline Género de los profesores & & & $\begin{array}{c}0.010 \\
(0.014)\end{array}$ & $\begin{array}{l}0.0315 \\
(0.013)\end{array}$ \\
\hline Nacionalidad & & & $\begin{array}{c}0.116^{* * * *} \\
(0.008)\end{array}$ & $\begin{array}{c}0.004 \\
(0.009)\end{array}$ \\
\hline Constante & $\begin{array}{c}1.062^{* * * *} \\
(0.010)\end{array}$ & $\begin{array}{c}1.148 * * * \\
(0.013)\end{array}$ & $\begin{array}{c}1.085^{* * * *} \\
(0.016)\end{array}$ & $\begin{array}{c}1.258 * * * \\
(0.017)\end{array}$ \\
\hline $\mathrm{R} 2$ & 0.365 & 0.367 & 0.380 & 0.438 \\
\hline Número de observaciones & 112 & 112 & 112 & 112 \\
\hline
\end{tabular}


Tabla 3. Regresión para la calidad educativa en Colombia 2016

\begin{tabular}{|c|c|c|c|c|}
\hline & & & & \\
\hline & Modelo 1 & Modelo 2 & Modelo 3 & Modelo 4 \\
\hline Formación de los docentes & $\begin{array}{c}-0.013^{* * *} \\
(0.001)\end{array}$ & $\begin{array}{c}-0.011 \text { *** } \\
(0.001)\end{array}$ & $\begin{array}{c}-0.017 * * * \\
(0.001)\end{array}$ & $\begin{array}{c}-0.009 \text { *** } \\
(0.001)\end{array}$ \\
\hline Tipo de universidad & $\begin{array}{c}-0.014^{* *} \\
(0.008)\end{array}$ & $\begin{array}{c}-0.039 * * * \\
(0.009)\end{array}$ & $\begin{array}{c}-0.026^{* * * *} \\
(0.008)\end{array}$ & $\begin{array}{c}-0.031^{* *} \\
(0.008)\end{array}$ \\
\hline Dedicación laboral & & $\begin{array}{c}-0.094 * * * \\
(0.020)\end{array}$ & & $\begin{array}{c}-0.065^{* * *} \\
(0.018)\end{array}$ \\
\hline Tipo de contrato & & $\begin{array}{c}-0.166^{* * * *} \\
(0.007)\end{array}$ & & $\begin{array}{c}-0.345^{\text {**** }} \\
(0.010)\end{array}$ \\
\hline Género de los profesores & & & $\begin{array}{l}0.009 \\
(0.031)\end{array}$ & $\begin{array}{c}0.075 * * \\
(0.025)\end{array}$ \\
\hline Nacionalidad & & & $\begin{array}{c}-0.107 * * * * \\
(0.009)\end{array}$ & $\begin{array}{l}-0.054 \\
(0.010)\end{array}$ \\
\hline Constante & $\begin{array}{c}2.167 * * * \\
(0.009)\end{array}$ & $\begin{array}{c}1.589 * * * \\
(0.047)\end{array}$ & $\begin{array}{c}1.334^{* * * *} \\
(0.030)\end{array}$ & $\begin{array}{c}1.610^{* * *} \\
(0.048)\end{array}$ \\
\hline $\mathrm{R} 2$ & 0.240 & 0.326 & 0.460 & 0.678 \\
\hline Número de observaciones & 112 & 112 & 112 & 112 \\
\hline
\end{tabular}

\section{Consideraciones finales}

En el presente documento se realizó un análisis de algunos aspectos pedagógicos que se deben tener en cuenta para ser aplicados al estudio de la economía y se llevó a cabo un análisis de literatura en torno al tema, tanto a nivel local como internacional. Posteriormente se realizó un análisis de hechos estilizados para buscar evidencia acerca de los efectos de las condiciones laborales y de formación de los docentes sobre la calidad educativa.

Desde el punto de vista metodológico, se planteó un modelo econométrico que buscaba medir el impacto de variables tales como nivel formación educativa de los docentes, tiempo de dedicación laboral, tipo de contrato, género y nacionalidad sobre la calidad educativa, medida a partir de los resultados de las pruebas Saber Pro.

En primer lugar, es importante destacar el papel que los acontecimientos ocurridos durante la última década del siglo XX han desempeñado en el orden económico mundial y cómo han establecido una nueva hoja de ruta para la docencia universitaria a nivel 
global. Dichos acontecimientos determinaron el inicio de una nueva etapa de globalización en la cual el conocimiento se encuentra en una esfera universal, marcando tendencias globales en la enseñanza y las prácticas pedagógicas. La utilización de nuevas tecnologías de la información y la comunicación (TIC) obliga a las instituciones de educación superior a actualizarse en línea con los nuevos retos que esta situación demanda.

Según la visión de algunos expertos, esta situación ha permitido que el avance de la sociedad se dé en términos desiguales, haciendo que las economías consideradas como más "débiles" terminen siendo las menos favorecidas en la competencia con las grandes economías por mantener e incrementar su poderío económico.

Por otra parte, desde el punto de vista conceptual, se logra observar que el estudio de la economía ha mostrado diversas tendencias en el siglo XXI, que van desde el enfoque convencional derivado del modelo neoclásico, hasta el retorno al pensamiento keynesiano, desarrollado por los pensadores norteamericanos y europeos a raíz de la crisis del capitalismo en 2007-2008.

En el ámbito pedagógico, la enseñanza de la economía se ha caracterizado por un marcado componente cuantitativo derivado de los modelos económicos actuales, que incorporan un alto nivel de rigurosidad para dar, en algunas ocasiones, soluciones y conclusiones bastante simples. Este nivel de formación ha sido ampliamente criticado por muchas corrientes de pensamiento y algunos expertos que consideran exagerado el uso de la formalización matemática en la elaboración de modelos económicos.

A pesar de esto, dichos modelos han sido de gran utilidad al momento de explicar problemáticas actuales y han sido referentes obligatorios por su nivel de precisión y análisis, lo cual alejaría la esfera de formalización del centro del debate para centrarla en el ámbito de su correcta aplicación e interpretación. Además de esto, en algunas ocasiones se emplean los modelos o teorías equivocados(as) para dar respuesta a las problemáticas actuales, mientras que otras veces se dan interpretaciones equivocadas a los modelos económicos.

Por otra parte, existe un segundo nivel de formación que parte del uso de instrumentos de tipo argumentativo y analítico para analizar la problemática económica a la que se enfrentan los seres humanos y su entorno. Este nivel de formación parte de visiones e interpretaciones subjetivas que llevan a la adopción de puntos de vista individuales basados en criterios propios. De esta situación se desprende la discrepancia entre los economistas y las diversas explicaciones y alternativas de solución que se dan al mismo problema.

De todas formas, en la actualidad, la ciencia económica ha logrado dar respuesta a las problemáticas actuales, e incluso en varias ocasiones ha sido un instrumento útil para predecir con un alto nivel de exactitud las crisis y recesiones ocurridas en los últimos 
tiempos. Es labor del docente de economía hacer comprender a los estudiantes que la ciencia económica, por tratarse de una ciencia social, no puede dar respuestas exactas a los acontecimientos y fenómenos ocurridos en este campo, pero que ello no la aleja de ser efectiva a la hora de explicarlos.

Trasciende la competencia de la ciencia económica el hecho de que los gobernantes y aquellos que toman decisiones en materia de política económica adopten y pongan en práctica todas las recomendaciones y conclusiones que los modelos y la teoría económica plantean con miras a alcanzar el objetivo básico de la economía: lograr la mejor manera de administrar los recursos escasos.

En cuanto a los resultados de las estimaciones realizadas, se puede concluir que tanto el nivel de formación como las condiciones laborales y el tipo de universidad inciden en la calidad educativa, medida a partir de los resultados de las pruebas Saber Pro. Específicamente, cuando el nivel de formación de los docentes es más alto, se observa una mejoría en los resultados de las pruebas. Esta situación se corrobora con los resultados obtenidos con respecto a las condiciones laborales. Contar con plantas profesorales en las cuales existan mejores condiciones contractuales puede arrojar mejores resultados en cuanto al logro de estudiantes con mayores competencias en el mercado laboral.

Por su parte, el género de los docentes no arrojó resultados estadísticamente significativos para demostrar la calidad académica. Por lo tanto, los resultados de las pruebas no están influidos por discriminaciones de sexo.

\section{Referencias}

Ahumada, I. y Butler, F. (marzo de 2009). La enseñanza de economía en México (Documento de trabajo n.o 672, BID). Washington: Banco Interamericano de Desarrollo (BID). Recuperado de goo.gl/umcdeG

Becker, W. E. y Watts, M. (2001). Teaching methods in U.S. Undergraduate Economics courses. The Fournal of Economic Education, 32(3), 269-279. Recuperado de goo.gl/ ePaqKe

Cataño, J. F. (2001). Discusión francesa sobre la enseñanza de la economía. Cuadernos de Economía, 20(35), 287-296. Recuperado de goo.gl/tg8ZTR

De Botssieu Christian, Jean-Jacques Rosa, Patrick Artus, Thierry Chauveau, Thierry Verdier, Georges Gallais-Hamonno, Pierre-Philippe Combes, Jean-Pau Betbèze, Claudia Sénik, Louis Lévy-Garboua, Antoine d 'Autume, Jean-Marc Daniel, Didier Marteau, Michel Didier, Alain Sand-Zantman. Crítica de profesores a la carta de los 
estudiantes. Publicado en: Discusión francesa sobre la enseñanza de la economía. Cuadernos de Economía, 20(35), 287-296. Recuperado de goo.gl/tg8ZTR

Dieterich, H. (1996). Globalización y educación: la ideología. Cuadernos de Economía, 15(25), 113-140. Recuperado de goo.gl/JeDqoU

Duran Silva, Luz Myriam (2006). Reflexiones sobre docencia universitaria para el siglo XX. Recuperado de https://revistas.lasalle.edu.co/index.php/ls/article/ viewFile/2096/1957

Gazzola, A. L. y Didriksson, A. (Eds.). (2008). Tendencias de la educación superior en América Latina y el Caribe. Caracas: IESALC-UNESCO.

Katz, C. (2001). El desafio crítico a los economistas ortodoxos [en línea]. Recuperado de goo. $\mathrm{gl} / \mathrm{BgVao} 4$

Lora, E. y Ñopo, H. (2009). La formación de los economistas en América Latina (Documento de trabajo n.o 119, BID). Washington: Banco Interamericano de Desarrollo.

Malamud, O. y Pop-Eleches, C. (2008). General education vs. Vocational training: Evidence from an economy in transition (Documento de trabajo n.o 14155, NBER). Cambridge: National Bureau of Economic Research. Recuperado de http://www.nber.org/ papers/w14155

Ortiz-Riaga, M. G. y Morales-Rubiano, M. E. (2011). La extensión universitaria en América Latina: concepciones y tendencias. Educación y Educadores, 14(2), 349-366. Recuperado de http://www.redalyc.org/articulo.oa?id=83421404008

Quintero, Marina; Giraldo Juan Leonel (2006). "La enseñanza: una práctica determinada por el deseo de saber". Diploma en fundamentación pedagógica y didáctica universitaria. Universidad de Antioquia, Medellín, pp. 81-89.

Rozenwurcel, G., Bezchinsky, G. L. y Rodríguez Chatruc, M. (2009). La enseñanza de economía en Argentina (Documento de trabajo n.o 671, BID). Washington: Banco Interamericano de Desarrollo. Recuperado de goo.gl/kgry0G

Salas, M. (2004). La relación educación-economía: un estudio del desajuste educativo de los titulados universitarios. Revista de Educación, 334, 259-278. Recuperado de goo. $\mathrm{gl} / \mathrm{xKNdqa}$

Sarmiento, J. A. y Silva, A. C. (2014). La formación del economista en Colombia. Revista Facultad de Ciencias Económicas: Investigación y Reflexión, 22(1), 231-262. Recuperado de http:/ /www.redalyc.org/articulo.oa?id=90931814015 


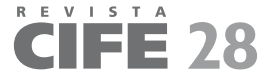

ISSN: 0124-3551 / Año 18, No 28 / enero-junio / pp. 19-42

Sfeir-Younis, A. (2009). Educación, economía ambiental y espiritualidad. Revista de Estudios Sociales, 32, 240-254. Recuperado de goo.gl/fHixGB

Tobón, A. (2008). Algunos elementos didácticos para la construcción de una propuesta pedagógica para la enseñanza de la economía. Recuperado de Apuntes del CENES, 27(46), 299-318. 